\title{
基于出行链的建成环境对居民小汽车 通勤出行的影响
}

\author{
张 雪 ${ }^{1,2}$, 周素红 ${ }^{1,2^{*}}$, 陈 菲 ${ }^{3}$ \\ (1. 中山大学地理科学与规划学院,广州 $510275 ; 2$. 广东省公共安全与灾害工程技术研究中心, 广州 510275 ; \\ 3. 中国城市规划设计研究院, 广东 深圳 518040)
}

\begin{abstract}
摘 要: 城市建成环境对居民出行, 尤其是小汽车通勤出行的影响是交通需求预测的基础性命题,长期受到关注。 已有研究大多以单次出行作为研究对象, 较少关注出行链影响下的出行协同决策问题。论文基于 2017 年广州市 居民活动日志调查数据, 采用Logistic 模型检验了出行链视角下城市建成环境对工作日选择小汽车通勤出行的影 响。研究发现, 出行链中的非通勤出行与职住地建成环境存在交互作用关系, 共同影响居民小汽车通勤出行方式 选择。一方面, 非通勤出行的时间顺序(到达就业地前的前项出行还是离开就业地后的后项出行)会影响职住地建成 环境对小汽车通勤出行方式选择的作用关系; 另一方面, 非通勤出行目的地的建成环境要素会调节职住地建成环境 与小汽车出行方式选择的关系。研究结论可为个体交通行为预测和基于个体模型的交通需求预测提供新的思路。
\end{abstract}

关 键 词: 建成环境; 小汽车通勤出行; 出行链; 交互作用

随着城市化进程的不断推进和城市交通的快 速发展, 小汽车通勤出行引发的高峰拥堵和环境污 染问题已经严重影响了居民的生活质量和城市的 可持续发展 ${ }^{[1-3]}$ 。通过建成环境引导可持续的交通 出行已成为共识, 国内外学者们对建成环境与居民 交通行为关系这一问题进行了大量研究和探索 ${ }^{[4-9]}$, 以期了解居民日常生活对城市建成环境相关要素 的空间配置需求, 为优化居民出行结构的城市交通 和城市规划提供参考。

建成环境与交通出行行为的相互作用关系是 长期的研究热点。对交通出行行为的关注包括出 行方式选择、出行距离、频率、时间和目的等。研究 中通常以密度(density)、混合度(diversity)、设计(design)、公交邻近度(distance to transit)、可达性(desti- nation accessibility) 5 个维度来对建成环境进行描 述 ${ }^{[10-11]}$ 。但是现有研究中对这几方面因素与出行行 为的影响关系仍未达成一致结论,大多数研究表明 建成环境要素会影响居民出行的频率、出行时间、 距离以及对出行交通方式的选择。在建成环境对 小汽车出行影响方面, 多数研究表明建筑密度、土 地利用混合度、商业可达性和公交站点对降低小汽 车使用的可能性, 而 POI 密度会增加居民使用小汽 车出行的比例 ${ }^{[12-13]}$ 。随着对地理背景不确定性问题 (uncertain geographic context problem, UGCoP)的讨 论不断增加, 研究者认识到基于传统的居住视角解 释行为的局限性。部分学者将工作地和其他活动 地的建成环境纳人讨论范围,认为忽视活动空间的 建成环境分析结果夸大了居住地对出行行为的影

收稿日期: 2020-06-17; 修订日期 : 2020-10-19。

基金项目: 国家自然科学基金项目(71961137003,41871148); 广东省重点领域研发计划项目(2020B0202010002)。[Foundation: National Natural Science Foundation of China, No. 71961137003 and 41871148; Key Research and Development Program of Guangdong Province, No. 2020B0202010002. ]

第一作者简介: 张雪(1992-),女,湖南娄底人,博士生,主要研究方向为时空行为、城市交通和健康地理。

E-mail: zhangx396@mail2.sysu.edu.cn

*通信作者简介: 周素红(1976-), 女,广东饶平人,教授,博士生导师,研究方向为城市地理学、城市交通、时空间行为、城市政 策与城乡规划。E-mail: eeszsh@mail.sysu.edu.cn

引用格式: 张雪, 周素红, 陈菲. 基于出行链的建成环境对居民小汽车通勤出行的影响 [J]. 地理科学进展, 2021, 40(4): 671-680. [Zhang Xue, Zhou Suhong, Chen Fei. Impact of the built environment on residents' car commuting based on trip chain. Progress in Geography, 2021, 40(4): 671-680. ] DOI: 10.18306/dlkxjz.2021.04.011 
响程度 ${ }^{[14-15]}$ 。

与此同时, 越来越多的学者认识到, 相比传统 的以单次出行作为研究单元(trip based), 基于出行 链(tour based)的分析能够更加准确地理解和预测出 行行为 ${ }^{[16-17]}$ 。出行链将出行者的所有活动通过出行 链接起来, 每日的出行过程都是多目的协同决策的 结果。在出行方式选择时, 通常会依据整日的出行 目的地来进行总体决策 ${ }^{[18-19]}$ 。尽管基于出行链的研 究可以更好地反映个体日常行为决策过程中的相 互关联关系, 但是关于建成环境对出行链行为影响 的共识还很少 ${ }^{[20-21]}$ 。部分研究表明, 建成环境要素 中的居住区位 ${ }^{[2]}$ 、居住地人口密度 ${ }^{[23]}$ 、建设密度 ${ }^{[24]}$ 、 土地利用混合度 ${ }^{[25]}$ 和可达性 ${ }^{[25]}$ 等对出行链行为(包 括出行链复杂性、出行方式选择、停靠次数、出行距 离等属性)有一定作用。

随着地理信息技术的不断发展,依托个体层面 海量信息数据指导和优化城市和交通规划已成为 新的趋势。因此, 如何将基于个体模型的建成环境 与交通出行研究成果运用到城市交通需求预测的 实际问题中也成为当前的关注重点。基于个体模 型的交通需求预测关键在于对个体出行行为与建 成环境关系的准确刻画, 以此推断个体在不同活动 需求和活动空间建成环境下选择各种交通方式出 行的概率, 从而预测不同活动空间的交通量。

城市建成环境对居民出行, 尤其是小汽车通勤 出行的影响是交通需求预测的基础性命题。然而, 对于出行链视角下建成环境对小汽车通勤出行方式 选择的影响还缺乏证据。尽管有研究表明, 通勤出 行过程中链接非工作出行可以减少小汽车总的行驶 里程和总行驶次数, 但随着出行链的复杂性增加, 越 来越多的人依赖小汽车来满足复杂的出行需求 ${ }^{[2]}$ 。 因此, 有必要从出行链的角度更加准确地刻画居民 小汽车通勤出行方式选择与建成环境的关系。

基于以上讨论, 本文提出几点假设: (1) 不同类 型的出行链中居民在小汽车通勤出行方式选择时 存在差异; (2) 出行链中非通勤出行对居民小汽车 通勤出行方式选择有一定的影响; (3) 非通勤出行 目的地建成环境对小汽车通勤出行方式选择也会 产生影响。为验证以上假设是否成立, 本文以居民 工作日多目的通勤出行链为研究对象, 通过构建二 项 Logistic 模型, 对比分析出行链视角下多目的地 出行及其建成环境对居民选择小汽车通勤出行的 交互影响。

\section{1 概念界定}

出行链是指将出行者从离开家再返回到家的 过程中所有的活动, 通过出行链接起来所形成的闭 合链 ${ }^{[17]}$ 。时空理论认为, 人不仅受到空间的约束, 也受到时间的约束。在出行链中, 以家和工作地为 场所的活动在地点和时间上相对固定。居民工作 日出行主要包括以家和工作地为针点的通勤出行, 以及以家和工作地为中心展开的其他出行。根据 出行链中的活动数量可以将其分为单链(只有工作 活动)和多链(有除工作以外的其他活动), 具体又可 分为在工作之前、在工作期间和在工作之后进行的 多链活动 ${ }^{[27]}$ 。本文主要针对通勤出行进行研究, 重 点关注上下班出行过程中有无其他活动, 具体总结 出 3 种不同形式的工作日通勤出行链。以 $\mathrm{H}$ 表示 家, $\mathrm{W}$ 表示工作地, $\mathrm{R}$ 表示其他活动地, 3 种出行链 表示为:

$\mathrm{H} \rightarrow \mathrm{W} \rightarrow \mathrm{H}$ : 只有工作活动的单链;

$\mathrm{H} \rightarrow \mathrm{R} \rightarrow \mathrm{W} \rightarrow \mathrm{H} / \mathrm{H} \rightarrow \mathrm{W} \rightarrow \mathrm{R} \rightarrow \mathrm{H}$ : 在上班/下班出 行过程中有其他活动的多链。当其他活动在工作 之前, 称之为通勤出行的前项活动, 当其他活动在 工作之后, 称之为通勤出行的后项活动;

$\mathrm{H} \rightarrow \mathrm{R} \rightarrow \mathrm{W} \rightarrow \mathrm{R} \rightarrow \mathrm{H}$ : 在上班和下班过程中都有 其他活动的多链。

相应地, 将以前项活动为目的的出行称之为前 项出行, 以后项活动为目的的出行称之为后项出 行,统称为非通勤出行。根据各出行段之间的时间 顺序组合关系, 当存在前项出行时, 工作活动的时 空棱镜受到前项活动地出发时间、工作开始时间, 以及从前项活动地到工作地的时间限制; 而存在后 项出行时，工作的结束时间、后项活动的开始时间、 从工作地到后项活动地的时间组成新的出行约 束。因此, 在后续的分析中, 将前项出行和后项出 行进行了区分并进行单独分析。

\section{2 研究区域、数据和方法}

\section{1 研究区域与数据来源}

选取了广州市内 5 种类型共 11 个街区进行调 研,分别位于广州市的天河、越秀、海珠、荔湾、白云 和番禺 6 个市辖区(图 1)。研究数据来源于 2017 年 6 月调查的广州市居民活动日志与社区融合问卷的 第一手资料。问卷总计回收 1003 份样本。选取居 


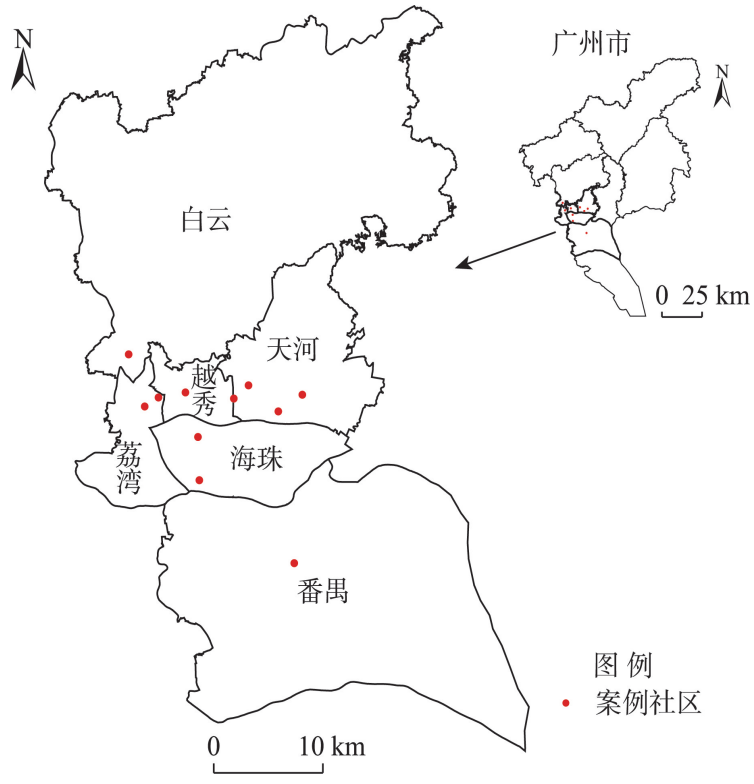

图 1 研究区域与案例社区分布

Fig.1 Location of the study area and the case communities

民工作日的出行日志数据, 去除无效出行和无出行 记录的样本, 最终剩下 982 位居民的 2547 条出行记 录, 人均日出行 2.91 次。问卷有效率为 $97.9 \%$ 。

有效问卷中男性样本量与女性样本量基本持 平。被调查者的年龄在 18 岁以上至 60 岁以下,其 中 19 30、31 45 岁分别均约占总样本量的 40\%。 $66.58 \%$ 的居民拥有中专及以上学历; $36.15 \%$ 的居民 拥有小汽车; $59.06 \%$ 的居民月收人在 4001 8000 元 之间, 20.06\%的居民月收人在 8000 元以上; 居民全 部就业。样本基本情况如表 1 所示。此外, 问卷出 行日志记录了居民一天的出行次数、出行起论点、 出行起止时间、出行同伴、出行方式、出行交通方式 调整性、活动类型、活动地点、活动同伴、活动起止 时间等信息。其中, 出行方式主要分为步行、单车 (私人单车和共享单车)、小汽车(驾驶或乘坐私人小 汽车)、出租车、公共汽车、地铁、单位班车, 文中将 出行方式归类为小汽车出行和非小汽车出行 2 类。 出行交通方式调整性主要采取主观评估的方法, 分 别设置了不可调整、较难调整、一般、较容易调整、 可随意调整 5 个选项，问卷中对应的选择为 1 5 分。 调整性越高, 说明居民对该种出行方式的依赖性越 小, 受出行方式的约束程度也越低。

\section{2 变量选择与模型构建}

根据以往研究结论, 出行者个体社会经济属性 和出行特性是影响居民选择小汽车出行的重要因 素 ${ }^{[28-30]}$ 。个体社会经济属性包括性别、年龄、教育水
表 1 被调查者社会经济属性

Tab.1 Individual socioeconomic attributes

\begin{tabular}{llcc}
\hline \multicolumn{1}{c}{ 变量 } & & 样本数 & 比例/\% \\
\hline 性别 & 男性 & 489 & 49.80 \\
& 女性 & 493 & 50.20 \\
年龄 & $19 \sim 30$ 岁 & 384 & 39.10 \\
& $31 \sim 45$ 岁 & 378 & 38.50 \\
& $46 \sim 59$ 岁 & 220 & 22.40 \\
教育 & 高中及以下 & 329 & 33.50 \\
& 大专或本科 & 644 & 65.58 \\
就业 & 研究生 & 9 & 0.92 \\
& 全职就业 & 975 & 99.29 \\
是否拥有小汽车 & 半职就业 & 7 & 0.71 \\
& 有 & 355 & 36.15 \\
月收人 & 无 & 627 & 63.85 \\
& 4000 元及以下 & 205 & 20.88 \\
& $4001 ~ 8000$ 元 & 580 & 59.06 \\
& $>8000$ 元 & 197 & 20.06 \\
\hline
\end{tabular}

平、就业、是否拥有小汽车、收人等; 出行特性包括 出行时间、是否有同伴同行等。本文在考虑出行者 个体属性和出行特性基础上, 重点探讨居住地、工 作地以及前后项出行目的地建成环境对小汽车通 勤出行的交互影响。建成环境包括建设密度、土地 利用混合度 ${ }^{[1]}$ 、交叉口密度、公交地铁密度和可达 性指标。数据的获取主要以居住地、工作地和其他 活动点地址为圆心, 分别做 $500 \mathrm{~m}$ 半径的缓冲区作 为地理背景统计单元。其中, 建设密度指标通过缓 冲区内的餐饮、教育、商业、休闲娱乐和医疗设施数 量总和除以缓冲区面积计算所得。交叉口密度是 以缓冲区内交叉口数量除以缓冲区面积。除此之 外, 基于广州市路网通过 ArcGIS 中的邻近度工具 计算每个点的可达性。

为回答前文中提出的假设,将其转化为具体的 研究问题, 并通过数据统计和构建模型进行分析。

问题一: 不同类型的出行链中居民选择小汽车 通勤出行的比例是否存在差异?

问题二: 有无前项/后项出行是否会调节职住地 建成环境与选择小汽车通勤出行的关系?

问题三: 前后项出行活动地建成环境是否会调 节职住地建成环境与选择小汽车通勤出行的关系?

为解答以上问题,数据分析工作分为以下 3 个 步骤: 首先, 对不同类型出行链中小汽车通勤出行 特征进行了描述性统计分析。其次,由于因变量 “选择小汽车通勤出行”为二分变量, 故采用二元 
Logistic 模型进行分析。通过加人 2 个变量的乘积 项表征交互效应 ${ }^{[32]}$, 以此来研究变量的调节作用, 交互模型如式(1) (7)所示。最后, 加人前后项出行 活动地建成环境与居住地/工作地建成环境的乘积 项, 进一步研究其他活动地建成环境对职住地建成 环境与选择小汽车通勤出行的调节作用, 模型如式 (8) (10) 所示。

$$
\begin{aligned}
& Z=B_{0}+\alpha_{0} C_{i}+\alpha_{1} H_{i}+\alpha_{2} W_{i}+\alpha_{3} X+ \\
& \beta_{1} H_{i} \times X+\beta_{2} W_{i} \times X+\varepsilon \\
& Z=B_{0}+\alpha_{0} C_{i}+\alpha_{1} H_{i}+\alpha_{2} W_{i}+\alpha_{4} Y+ \\
& \beta_{3} H_{i} \times Y+\beta_{4} W_{i} \times Y+\varepsilon \\
& C_{i}=\left[\begin{array}{llll}
C_{1 i} & C_{2 i} & \cdots & C_{m i}
\end{array}\right] \\
& H_{i}=\left[\begin{array}{llll}
H_{1 i} & H_{2 i} & \cdots & H_{n i}
\end{array}\right] \\
& W_{i}=\left[\begin{array}{llll}
W_{1 i} & W_{2 i} & \cdots & W_{n i}
\end{array}\right] \\
& X= \begin{cases}0 & \text { 无前项出行 } \\
1 & \text { 有前项出行 }\end{cases} \\
& Y= \begin{cases}0 & \text { 无后项出行 } \\
1 & \text { 有后项出行 }\end{cases} \\
& Z=B_{0}+\alpha_{0} C_{i}+\alpha_{1} H_{i}+\alpha_{5} R_{i}+\beta_{5} H_{i} \times R_{i}+\varepsilon \\
& Z=B_{0}+\alpha_{0} C_{i}+\alpha_{2} W_{i}+\alpha_{5} R_{i}+\beta_{6} W_{i} \times R_{i}+\varepsilon \\
& R_{i}=\left[\begin{array}{llll}
R_{1 i} & R_{2 i} & \cdots & R_{n i}
\end{array}\right]
\end{aligned}
$$

式中: $Z$ 为选择小汽车出行的虚拟变量, $Z=1$ 表示 使用小汽车出行, $Z=0$ 表示没有使用小汽车出行; $B_{0}$ 为常数项, $\varepsilon$ 为误差项, $i$ 为样本数量; $C_{i}$ 表示个 人属性变量和出行特性变量, 共有 $m$ 项; $H_{i}$ 表示居 住地建成环境变量集, $W_{i}$ 表示工作地建成环境变 量集, $R_{i}$ 表示其他活动地建成环境变量集(前项活 动表示为 $F_{i}$, 后项表示为 $L_{i}$ ), 建成环境又分别包 括建设密度、土地利用混合度、交叉口密度、公交地 铁密度和可达性 5 项, 即 $n=5 ; X 、 Y$ 分别为有无前 项出行和后项出行的虚拟变量; $\alpha_{0} 、 \alpha_{1} 、 \alpha_{2}$ 分别为 个体属性变量和出行特性变量、居住地环境变量 集、工作地环境变量集的回归系数; $\alpha_{3} 、 \alpha_{4}$ 为有无 前项出行、有无后项出行变量的回归系数; $\alpha_{5}$ 为其
他活动地环境变量集的回归系数; $\beta_{1} \sim \beta_{6}$ 分别对应 各交互项的回归系数。所有连续变量均经过 $Z$ score标准化处理。

\section{3 基于出行链的小汽车通勤出行特征}

根据数据统计, 除了以往多数研究关注的往返 于家和工作地之间的简单出行链模式外, 多目的出 行链现象同样不容忽视。在出行模式比例方面, 工 作日 $\mathrm{H} \rightarrow \mathrm{W} \rightarrow \mathrm{H}$ 模式的出行链样本比例为 $55.60 \%$, $\mathrm{H} \rightarrow \mathrm{R} \rightarrow \mathrm{W} \rightarrow \mathrm{H}$ 与 $\mathrm{H} \rightarrow \mathrm{W} \rightarrow \mathrm{R} \rightarrow \mathrm{H}$ 模式的出行链样本 比例分别为 $5.19 \%$ 和 $36.25 \%, \mathrm{H} \rightarrow \mathrm{R} \rightarrow \mathrm{W} \rightarrow \mathrm{R} \rightarrow \mathrm{H}$ 模 式的出行链样本比例为 $2.95 \%$ (表 2)。说明近一半 的居民工作日出行活动模式较为复杂, 除工作为目 的的通勤出行外还有其他活动的出行, 并且大多为 下班后的活动出行。在分析工作日小汽车通勤出 行行为时不仅需要考虑到居住地和工作地建成环 境的影响, 还需从出行链的整体性视角出发, 考虑 其他活动对通勤出行的间接影响关系。

在不同类型出行链中使用小汽车通勤出行的 人数方面, $\mathrm{H} \rightarrow \mathrm{W} \rightarrow \mathrm{H}$ 单链中使用小汽车通勤出行 的人数在总的单链中占比为 $12.09 \%$, 而多链 $\mathrm{H} \rightarrow$ $\mathrm{W} \rightarrow \mathrm{R} \rightarrow \mathrm{H}$ 和 $\mathrm{H} \rightarrow \mathrm{R} \rightarrow \mathrm{W} \rightarrow \mathrm{R} \rightarrow \mathrm{H}$ 中使用小汽车通勤 出行的人数占比分别为 $12.92 \%$ 和 $13.79 \%$ 。可见,

\begin{tabular}{|c|c|c|c|c|}
\hline \multirow{2}{*}{ 出行链 } & \multicolumn{2}{|c|}{ 总样本 } & \multicolumn{2}{|c|}{ 使用小汽车通勤出行样本 } \\
\hline & 数量/人 & 比例/\% & 数量/人 & 在总样本中占比 $/ \%$ \\
\hline $\mathrm{H} \rightarrow \mathrm{W} \rightarrow \mathrm{H}$ & 546 & 55.60 & 66 & 12.09 \\
\hline $\mathrm{H} \rightarrow \mathrm{R} \rightarrow \mathrm{W} \rightarrow \mathrm{H}$ & 51 & 5.20 & 4 & 7.84 \\
\hline $\mathrm{H} \rightarrow \mathrm{W} \rightarrow \mathrm{R} \rightarrow \mathrm{H}$ & 356 & 36.25 & 46 & 12.92 \\
\hline $\mathrm{H} \rightarrow \mathrm{R} \rightarrow \mathrm{W} \rightarrow \mathrm{R} \rightarrow \mathrm{H}$ & 29 & 2.95 & 4 & 13.79 \\
\hline 总计 & 982 & 100 & 120 & 12.22 \\
\hline
\end{tabular}
不同类型的出行链中选择小汽车通勤出行的比例 有一定差异, 出行链越复杂, 选择小汽车通勤出行 的可能性越高, 回答了问题一。但在有前项出行的 $\mathrm{H} \rightarrow \mathrm{R} \rightarrow \mathrm{W} \rightarrow \mathrm{H}$ 类型中, 选择使用小汽车通勤出行的 比例相比单链来说更低。因此, 对于多链中有无前 后项出行对居民选择小汽车通勤出行的影响关系 还需进一步分析。

\section{4 模型结果与分析}

由前文的分析可知, 55.60\%的居民只有以工作

表 2 工作日出行链特征

Tab.2 Characteristics of trip chain on weekdays 
为目的的通勤出行, $44.40 \%$ 的居民有除工作以外的 其他活动的出行。因此, 在 $\mathrm{H} \rightarrow \mathrm{R} \rightarrow \mathrm{W} \rightarrow \mathrm{H} 、 \mathrm{H} \rightarrow$ $\mathrm{W} \rightarrow \mathrm{R} \rightarrow \mathrm{H} 、 \mathrm{H} \rightarrow \mathrm{R} \rightarrow \mathrm{W} \rightarrow \mathrm{R} \rightarrow \mathrm{H}$ 三种出行链模式中, 需要将非通勤出行及其活动地建成环境的影响也 纳人分析。为保持二项 Logistic 回归模型中交互项 量纲的一致性, 对出行链中出现多次前项或后项出 行的情况, 根据出行目的地的 “活动地点可调整性” 和“活动时间可调整性”指标,最后选取时空可调性 最小(时空制约性最大)的活动地纳人模型分析中, 分别以 $\mathrm{F}$ 和 $\mathrm{L}$ 表示前、后项活动地建成环境。对于 同时有前后项活动地的出行链, 由于样本量较少 $(\mathrm{H} \rightarrow \mathrm{R} \rightarrow \mathrm{W} \rightarrow \mathrm{R} \rightarrow \mathrm{H}$ 类型占比 $2.95 \%)$, 将其分别归人 有前项活动和有后项活动 2 种情况, 不再单独考虑。

对所构建的二项 Logistic 回归模型进行拟合， 以模型 1 只考虑居民个体属性和出行特性的影响作 为参照, 模型 $2 、 3 、 4$ 分别加人了居住地、工作地、居 住地和工作地建成环境属性, 以考察居住地、工作 地建成环境及其两者同时对居民选择小汽车通勤 出行的影响。在此基础上, 分别计算了有无前项出 行和后项出行与居住地和工作地建成环境的交互 项, 并将其分别加人模型 5、6 中, 以探讨多链中非通 勤出行的时间顺序对职住地建成环境与居民选择 小汽车通勤出行关系的影响。通过逐步回归,最终 进人回归模型的变量以及各模型拟合结果如表 3 所 示。在此基础上,进一步探讨了非通勤出行目的地 建成环境对小汽车通勤出行可能产生的影响(表 4)。

\section{1 职住地建成环境与小汽车通勤出行的关系}

表 3 中模型 2 4 分析了职住地建成环境对居民 选择小汽车通勤出行的影响。结果表明, 居住地建 成环境对小汽车通勤出行有显著影响。其中, 居住 地土地利用混合度与选择小汽车通勤出行呈显著 的负相关关系, 说明居住地土地利用混合度的提高 可以显著减少小汽车在通勤出行中的使用, 这与西 方已有的研究结论一致 ${ }^{[15]}$ 。但仅考虑居住地建成 环境对小汽车通勤出行影响的模型未能通过显著 性检验(模型 2, Sig $>0.1$ )。而工作地建成环境对小 汽车通勤出行无明显的影响(模型 3$)$ 。同时考虑了 居住地和工作地建成环境后, 居住地土地利用混合 度对小汽车通勤方式选择影响显著, 且模型通过显 著性检验(模型 4)。

\section{2 非通勤出行对职住地建成环境与小汽车通勤出 行关系的调节作用}

(1) 非通勤出行时间顺序的调节作用

模型 5 分析了前项出行对职住地建成环境与小
汽车通勤出行的调节作用。可以发现, 当有前项出 行时, 模型 5 中居住地土地利用混合度与小汽车通 勤出行仍然呈显著的负相关关系。此外, 交互项 “ $H$ - 可达性 $\times X$ ” 有显著相关性, 回归系数为 2.540 , 通 过计算其系数指数值为 12.678 。表明当居住地可 达性增加时, 相比无前项出行, 有前项出行时居民 选择小汽车通勤的概率会增长至12.678 倍。可见， 有前项出行时，居住地可达性的提高会促使居民选 择小汽车通勤出行的比例大幅增加。这也说明了 有前项出行对居住地可达性与小汽车通勤出行方 式选择有显著的调节作用。

模型 6 分析了后项出行对职住地建成环境与小 汽车通勤出行的调节作用。可以发现, 考虑有后项 出行后, 职住地的各项建成环境指标与小汽车通勤 出行并无显著的相关关系, 并且后项出行与职住地 建成环境的交互项也不显著。原因可能在于后项 地的加人削弱或剥夺了职住地建成环境变量的影 响,其作用机制还需进一步探讨。

综上, 模型结果表明了前项出行和后项出行对 职住地建成环境与小汽车通勤出行两者关系的调 节作用, 回答了问题二。前项出行主要通过调节居 住地可达性对小汽车通勤出行方式选择产生影响, 后项出行对职住地建成环境与小汽车通勤的关系 没有显著的调节作用。但是当有后项出行时, 职住 地建成环境对小汽车通勤出行的影响都不再显著, 具体的影响机制还需进一步探讨。

(2) 非通勤出行目的地建成环境的调节作用

为进一步揭示后项出行与职住地建成环境的 交互作用关系, 以有后项出行的 385 个样本构建后 项出行目的地建成环境与职住地建成环境的交互 模型。回归模型均显著, 结果如表 4 。

从后项出行目的地建成环境与居住地建成环 境的交互影响结果可以发现,后项活动地交叉口密 度对小汽车通勤出行有显著的影响, 回归系数为 0.184 , 系数指数值为 1.202 。说明后项活动地的交 叉路口密度的提高, 会使选择小汽车通勤出行的概 率显著增加, 这与以往基于居住地交叉路口研究的 结论恰好相反 ${ }^{[33-34]}$ 。原因可能在于, 较高的交叉口 密度意味着较高的交通通达性, 通常意义上认为会 增加非机动出行的概率。但是当活动增加、出行链 变得复杂时,活动地较高的交通通达性可能会导致 选择小汽车出行比例的增加。因为即使有较为便 利的公共交通,但是活动增加意味着交通换乘的次 数也在增加, 这反而为出行带来不便。之前的研究 
表 3 工作日小汽车通勤出行方式选择的二元 Logistic 模型结果

Tab.3 Binary Logistic regression of the car commuting on weekdays

\begin{tabular}{|c|c|c|c|c|c|c|c|}
\hline \multirow{2}{*}{\multicolumn{2}{|c|}{ 变量 }} & \multicolumn{4}{|c|}{ 未考虑其他活动出行 } & \multicolumn{2}{|c|}{ 考虑其他活动出行 } \\
\hline & & 模型 1 & \multirow{2}{*}{$\begin{array}{c}\begin{array}{c}\text { 模型 } 2 \\
\text { (居住地) }\end{array} \\
-5.610^{* * *}\end{array}$} & \multirow{2}{*}{$\begin{array}{c}\begin{array}{c}\text { 模型 } 3 \\
\text { (工作地) }\end{array} \\
-5.267^{* * *}\end{array}$} & $\begin{array}{c}\text { 模型 } 4 \\
\text { (居住地和工作地) }\end{array}$ & $\begin{array}{c}\text { 模型 } 5 \\
\text { (前项出行交互作用) }\end{array}$ & $\begin{array}{c}\text { 模型 } 6 \\
\text { (后项出行交互作用) }\end{array}$ \\
\hline 常数项 & & $-5.237^{* *}$ & & & $-5.322^{* * *}$ & $-5.305^{* * *}$ & $-8.454^{* * * *}$ \\
\hline \multicolumn{8}{|l|}{ 社会经济属性 } \\
\hline \multirow[t]{2}{*}{ 年龄 } & $31 \sim 45$ 岁 & $0.956^{* *}$ & $0.963^{* *}$ & $0.999^{* *}$ & $1.222^{* *}$ & $1.223^{* * *}$ & $1.268^{* *}$ \\
\hline & 46 59 岁 & -0.421 & -0.287 & -0.313 & -0.012 & -0.082 & -0.217 \\
\hline 是否有小汽车 & 有 & $3.181^{* * * *}$ & $3.392^{* * *}$ & $3.241^{* * *}$ & $3.360^{* * *}$ & $3.465^{* * *}$ & $3.551^{* * * *}$ \\
\hline \multirow[t]{2}{*}{ 月收人 } & $4001 \sim 8000$ 元 & $1.872^{* *}$ & $1.602^{* *}$ & $1.811^{* *}$ & $1.356^{\circ}$ & $1.374^{*}$ & $1.525^{*}$ \\
\hline & $>8000$ 元 & $2.576^{* * * *}$ & $2.195^{* * *}$ & $2.388^{* * * *}$ & $1.954^{* *}$ & $2.007^{* *}$ & $2.173^{* *}$ \\
\hline \multicolumn{8}{|l|}{ 出行特性 } \\
\hline 有无出行同伴 & & $2.250^{* * * *}$ & $2.163^{* * *}$ & $2.292^{* * *}$ & $2.036^{* * *}$ & $2.402^{* * * *}$ & $2.507^{* * *}$ \\
\hline 出行时间 & & $-1.926^{* * *}$ & $-1.649^{* *}$ & $-1.910^{* * * *}$ & $-1.958^{* * * *}$ & $-2.187^{* * * *}$ & $-2.086^{* * *}$ \\
\hline$X$ & & & & & & -3.021 & \\
\hline$Y$ & & & & & & & 2.850 \\
\hline \multicolumn{8}{|l|}{ 建成环境 } \\
\hline \multicolumn{2}{|c|}{ H_土地利用混合度 } & & $-0.794^{* *}$ & & $-0.721^{* *}$ & $-0.782^{* *}$ & -0.196 \\
\hline \multicolumn{2}{|l|}{$H_{-}$建设密度 } & & 0.381 & & 0.271 & 0.273 & -2.690 \\
\hline \multicolumn{2}{|l|}{$H$ _交叉口密度 } & & -0.192 & & -0.339 & -0.061 & -1.139 \\
\hline \multicolumn{2}{|l|}{$H_{-}$可达性 } & & -0.089 & & -0.134 & -0.402 & 3.157 \\
\hline \multicolumn{2}{|c|}{$W_{-}$土地利用混合度 } & & & -0.171 & -0.193 & -0.195 & -0.503 \\
\hline \multicolumn{2}{|l|}{$W_{-}$建设密度 } & & & -0.205 & 0.128 & 0.041 & 1.430 \\
\hline \multicolumn{2}{|l|}{$W$ _交叉口密度 } & & & 0.198 & 0.262 & 0.411 & -4.609 \\
\hline \multicolumn{2}{|l|}{$W_{-}$可达性 } & & & 0.069 & 0.041 & 0.219 & 3.052 \\
\hline \multicolumn{2}{|c|}{$H \_$土地利用混合度 $\times X$} & & & & & -0.581 & \\
\hline \multicolumn{2}{|l|}{$H$ 建设密度 $\times X$} & & & & & 1.925 & \\
\hline$H_{-}$交叉口密度 $\times$ & & & & & & -5.322 & \\
\hline$H_{\text {_ 可达性 } \times X}$ & & & & & & $2.540^{*}$ & \\
\hline$W_{-}$土地利用混合 & 合度 $\times X$ & & & & & -0.816 & \\
\hline$W_{-}$建设密度 $\times X$ & & & & & & 1.175 & \\
\hline$W$ 交叉口密度 $\times$ & & & & & & -1.440 & \\
\hline$W_{\text {_ 可达性 } \times X}$ & & & & & & -0.757 & \\
\hline$H_{-}$土地利用混合 & 合度 $\times Y$ & & & & & & -0.767 \\
\hline$H$ _建设密度 $\times Y$ & & & & & & & 3.016 \\
\hline$H_{-}$交叉口密度 $\times$ & & & & & & & 1.377 \\
\hline$H_{-}$可达性 $\times Y$ & & & & & & & -3.653 \\
\hline$W_{\text {_ 土地利用混合 }}$ & 合度 $\times Y$ & & & & & & 0.170 \\
\hline$W_{-}$建设密度 $\times Y$ & & & & & & & -1.569 \\
\hline$W$ 交叉口密度 $\times$ & & & & & & & 4.881 \\
\hline$W_{-}$可达性 $\times Y$ & & & & & & & -2.632 \\
\hline 伪 $R^{2}$ & & 0.529 & 0.571 & 0.539 & 0.583 & 0.621 & 0.619 \\
\hline-2 log likelihood & & 183.96 & 170.54 & 180.70 & 165.55 & 153.54 & 154.04 \\
\hline$P$ & & 0.001 & 0.103 & $<0.001$ & 0.014 & $<0.001$ & 0.013 \\
\hline
\end{tabular}

注: ***、**、分别表示在 $0.01 、 0.05 、 0.1$ 显著性水平下显著。下同。 
表 4 后项出行目的地建成环境交互影响回归模型结果

Tab.4 Regression results of the built environment and after work trip interaction

\begin{tabular}{|c|c|c|c|c|c|}
\hline \multicolumn{3}{|c|}{ 居住地交互影响 } & \multicolumn{3}{|c|}{ 工作地交互影响 } \\
\hline \multicolumn{2}{|l|}{ 变量 } & \multirow{2}{*}{$\frac{\text { 系数 }}{-6.049^{* *}}$} & \multicolumn{2}{|c|}{ 变量 } & \multirow{2}{*}{$\frac{\text { 系数 }}{-5.306^{* * *}}$} \\
\hline 常数项 & & & 常数项 & & \\
\hline \multirow[t]{2}{*}{ 年龄 } & $31 \sim 5$ 岁 & $0.945^{*}$ & 年龄 & $31 \sim 45$ 岁 & $1.259^{* *}$ \\
\hline & 46 59 岁 & -0.537 & & 46 59 岁 & -0.323 \\
\hline 是否有小汽车 & 有 & $3.508^{* * * *}$ & 是否有小汽车 & 有 & $3.272^{* * *}$ \\
\hline \multirow[t]{2}{*}{ 月收人(元) } & $4001 \sim 8000$ 元 & $1.831^{* * *}$ & 月收人 & $4001 \sim 8000$ 元 & $1.854^{* *}$ \\
\hline & $>8000$ 元 & $2.175^{* * *}$ & & $>8000$ 元 & $2.215^{* * *}$ \\
\hline 有无出行同伴 & 有 & $2.666^{* * *}$ & 有无出行同伴 & 有 & $2.639^{* * *}$ \\
\hline 出行时间 & & $-1.532^{*}$ & 出行时间 & & $-2.166^{* * * *}$ \\
\hline H_土地利用混合度 & & $-1.006^{* * *}$ & $W$ _土地利用混 & & -0.245 \\
\hline$H$ _建设密度 & & 0.437 & $W$ _建设密度 & & 0.166 \\
\hline$H$ _交叉口密度 & & -0.385 & $W$ _交叉口密度 & & 0.329 \\
\hline$H_{-}$可达性 & & -0.184 & $W_{-}$可达性 & & 0.054 \\
\hline$L_{-}$土地利用混合度 & & -0.044 & $L_{-}$土地利用混 & & -0.021 \\
\hline L_建设密度 & & -0.012 & L_建设密度 & & 0.006 \\
\hline$L$ _交叉口密度 & & $0.184^{*}$ & $L$ _交叉口密度 & & 0.033 \\
\hline$L_{\text {_可达性 }}$ & & -0.065 & $L_{-}$可达性 & & 0.007 \\
\hline$H \times L_{-}$土地利用混合度 & & 0.022 & $W \times L \_$土地利用 & & -0.125 \\
\hline$H \times L \_$建设密度 & & -0.109 & $W \times L \_$建设密度 & & $-1.050^{*}$ \\
\hline$H \times L_{-}$交叉口密度 & & 0.677 & $W \times L$ _交叉口密 & & -0.447 \\
\hline$H \times L_{-}$可达性 & & 0.011 & $W \times L_{-}$可达性 & & 0.756 \\
\hline 伪 $R^{2}$ & & 0.622 & 伪 $R^{2}$ & & 0.592 \\
\hline-2 log likelihood & & 140.42 & -2 log likelihoo & & 149.60 \\
\hline Sig & & 0.011 & Sig & & $<0.001$ \\
\hline
\end{tabular}

也表明下班后产生的活动需求催生了更多的复杂 出行链,使通勤者更多选择小汽车出行 ${ }^{[35]}$ 。

从后项出行目的地建成环境与工作地建成环 境的交互影响结果可以发现, 后项活动地建设密度 和工作地建设密度的交互项与小汽车通勤出行呈 显著负相关, 相关系数为 -1.050 , 系数指数为 0.350 。说明后项活动地建设密度增加时,工作地建 设密度对小汽车通勤出行的影响会下降 $[\exp (W$ 建 设密度 $) \times 0.350<\exp \left(W_{-}\right.$建设密度 $\left.)\right]$。可见, 后项活 动地建成环境对工作地建成环境与通勤出行方式 选择的影响关系有一定的拮抗作用。模型结果也 回答了问题三。

综上, 居民在小汽车通勤出行行为选择时, 出 行链上的非通勤出行(即前项和后项出行)与职住地 建成环境具有交互作用关系。一方面, 非通勤出行 的时间顺序(前项出行还是后项出行)会影响职住地 建成环境对小汽车通勤出行的作用关系; 另一方 面, 非通勤出行尤其是后项出行目的地建成环境与
工作地建成环境也存在交互作用关系, 从而影响小 汽车通勤出行方式选择。

\section{5 结论与讨论}

\section{1 结论}

本文基于广州市的实证研究,尝试从出行链视 角分析建成环境对小汽车通勤出行的影响。在对 居民工作日出行链特征分析的基础上,进一步探讨 了非通勤出行及其目的地建成环境对小汽车通勤 出行的影响关系。研究比较了复杂出行链下非通 勤出行的时空要素(非通勤出行的时间顺序和非通 勤出行目的地建成环境要素)与职住地建成环境对 出行者选择小汽车通勤出行的协同作用关系。

研究结论主要包括: (1) $44.40 \%$ 的居民在工作 日有除 $\mathrm{H} \rightarrow \mathrm{W} \rightarrow \mathrm{H}$ 单链条出行活动以外的出行链, 仅从居住地和工作地的建成环境分析居民通勤出 行行为, 其解释力度有限, 并且不同类型的通勤链 
中居民选择小汽车通勤出行的比例存在一定差异, 假设一成立。(2) 出行链上的非通勤出行对职住地 建成环境与小汽车通勤出行的关系有调节作用, 并 且这种调节作用因非通勤出行的时间顺序而不一 致(前项出行主要通过调节居住地可达性对小汽车 通勤出行产生影响, 后项出行对职住地建成环境与 小汽车通勤的关系没有显著的调节作用), 假设二 成立。(3) 出行链中非通勤出行目的地建成环境对 小汽车通勤出行的选择有一定解释力, 更为真实地 揭示了小汽车通勤出行方式选择时考虑到的实际 地理背景, 能够捕捉到一些重要的影响因素。例如 非通勤出行后项活动地的交叉口密度会显著影响 小汽车通勤出行的选择, 以及后项活动地的建设密 度对小汽车出行也会有一定的调节作用关系, 假设 三成立。(4) 研究结论也对以往研究结果的矛盾之 处有一定的解释力, 一方面, 地理背景界定存在差 异会导致研究结论的不一致; 另一方面, 真实的出 行方式选择需要将所有的出行作为整体进行考虑, 而不是将单趟出行作为研究对象, 否则会导致研究 结果的偏颇。

\section{2 讨论}

本文基于广州市的案例, 探讨了出行链视角下 多目的地出行及其建成环境对居民选择小汽车通 勤出行的交互影响。一方面, 研究论证了出行链对 小汽车通勤出行的制约关系; 另一方面, 研究结果 也为个体交通行为预测和基于个体模型的交通需 求预测提供新的思路。传统的小汽车出行选择模 型和出行链类型决策模型大多考虑了距离衰减和 自选择偏好, 而本文研究结果表明出行链上其他活 动地的建成环境对个体选择小汽车出行也有一定 的影响, 研究结论为地理背景的不确定性问题(UG$\mathrm{CoP}$ ) 提供了新的证据。此研究结果潜在的应用价 值在于, 利用信息技术获取海量个体时空轨迹数据 的同时, 依据出行链视角下个体小汽车通勤出行方 式选择与建成环境的影响关系, 可以建立更加精确 的个体小汽车出行行为预测规则, 从而实现对城市 早晚高峰交通需求和不同活动空间出行量的预测, 进而指导城市交通规划和政策的制定。

当然, 针对出行链视角下建成环境对居民选择 小汽车通勤出行的影响这一问题, 还有值得进一步 讨论的地方。首先, 在模型中有多个建成环境指标 并不显著, 例如建设密度、交叉口密度等, 虽然以往 研究结论不一致 ${ }^{[4,36-37]}$, 也有国外研究表明居住地建
成环境对居民出行方式选择的影响并不明显,但对 变量间的交互关系以及多目的地建成环境变量间 的交互关系还需进一步研究。本文虽然发现部分 建成环境指标间具有显著的交互关系,但是由于数 据样本限制, 未考虑前项活动地与职住地建成环境 的作用关系, 也未考虑同时有前后项出行时的三项 协同等更为复杂的情况。此外,在研究其他非通勤 出行对小汽车通勤出行方式选择的影响时, 并未将 每趟出行都考虑进来, 与实际情况仍有一些差异。 最后,基于出行链的通勤出行研究虽然能够探测更 多的出行方式选择影响因素,但是与单趟出行研究 相比,需要控制和考虑的因素也更多, 对研究数据 的精度要求更高。传统问卷调查在获取细节化信 息的同时,也可能因个人对出行链的记忆偏差和遗 漏而导致结果存在偏差的风险。

\section{参考文献(References)}

[1] 杨文越, 梁斐雯, 曹小曙. 多尺度建成环境对居民通勤出 行碳排放的影响: 来自广州的实证研究 [J]. 地理研究, 2020, 39(7): 1625-1639. [Yang Wenyue, Liang Feiwen, Cao Xiaoshu. Examining the effects of the multi-scale built environment on residents' $\mathrm{CO}_{2}$ emissions from commuting: An empirical study of Guangzhou. Geographical Research, 2020, 39(7): 1625-1639. ]

[2] 杨文越, 曹小曙. 多尺度交通出行碳排放影响因素研究 进展 [J]. 地理科学进展, 2019, 38(11): 1814-1828. [Yang Wenyue, Cao Xiaoshu. Progress of research on influencing factors of $\mathrm{CO}_{2}$ emissions from multi-scale transport. Progress in Geography, 2019, 38(11): 1814-1828. ]

[3] 马静, 柴彦威, 符婷婷. 居民时空行为与环境污染暴露对 健康影响的研究进展 [J]. 地理科学进展, 2017, 36(10): 1260-1269. [Ma Jing, Chai Yanwei, Fu Tingting. Progress of research on the health impact of people's space-time behavior and environmental pollution exposure. Progress in Geography, 2017, 36(10): 1260-1269. ]

[4] 陈博文, 周素红, 姜超. 活动视角下建成环境对广州居民 通勤效率的影响 [J]. 城市规划学刊, 2016(4): 67-74. [Chen Bowen, Zhou Suhong, Jiang Chao. The impacts of built environment on residents' commuting efficiency based on activities in Guangzhou. Urban Planning Forum, 2016(4): 67-74. ]

[5] 曹新宇. 社区建成环境和交通行为研究回顾与展望: 以 美国为鉴 [J]. 国际城市规划, 2015, 30(4): 46-52. [Cao Xinyu. Examining the relationship between neighborhood built environment and travel behavior: A review from the US perspective. Urban Planning International, 2015, 30 
(4): 46-52. ]

[6] Wang D G, Chai Y W, Li F. Built environment diversities and activity-travel behavior variations in Beijing, China [J]. Journal of Transport Geography, 2011, 19(6): 11731186.

[7] Cervero R. Built environments and mode choice: Toward a normative framework [J]. Transportation Research Part D: Transport and Environment, 2002, 7(4): 265-284.

[8] Ewing R, Cervero R. Travel and the built environment: A synthesis [J]. Transportation Research Record: Journal of the Transportation Research Board, 2001, 1780: 87-114.

[9] Wang D G, Zhou M. The built environment and travel behavior in urban China: A literature review [J]. Transportation Research Part D: Transport and Environment, 2017, 52: 574-585.

[10] Cervero R, Kockelman K. Travel demand and the 3Ds: Density, diversity, and design [J]. Transportation Research Part D: Transport and Environment, 1997, 2(3): 199-219.

[11] Ewing R, Cervero R. Travel and the built environment [J]. Journal of the American Planning Association, 2010, 76(3): 265-294.

[12] Khattak A J, Rodriguez D. Travel behavior in neo-traditional neighborhood developments: A case study in USA [J]. Transportation Research Part A: Policy and Practice, 2005, 39(6): 481-500.

[13] 周素红, 宋江宇, 宋广文. 广州市居民工作日小汽车出 行个体与社区双层影响机制 [J]. 地理学报, 2017, 72 (8): 1444- 1457. [Zhou Suhong, Song Jiangyu, Song Guangwen. Examining the dual-levels impact of neighborhood and individual variables on car use on weekdays in Guangzhou. Acta Geographica Sinica, 2017, 72(8): 1444-1457. ]

[14] Jones M, Pebley A R. Redefining neighborhoods using common destinations: Social characteristics of activity spaces and home census tracts compared [J]. Demography, 2014, 51(3): 727-752.

[15] 塔娜, 柴彦威, 关美宝. 建成环境对北京市郊区居民工 作日汽车出行的影响 [J]. 地理学报, 2015, 70(10): 1675-1685. [Ta Na, Chai Yanwei, Kwan Mei-po. The relationship between the built environment and car travel distance on weekdays in Beijing. Acta Geographica Sinica, 2015, 70(10): 1675-1685. ]

[16] Currie G, Delbosc A. Exploring the trip chaining behavior of public transport users in Melbourne [J]. Transport Policy, 2011, 18(1): 204-210.

[17] Primerano F, Taylor M A P, Pitaksringkarn L, et al. Defining and understanding trip chaining behaviour [J]. Transportation, 2008, 35(1): 55-72.
[18] 奕琨, 隽志才, 宗芳. 通勤者出行方式与出行链选择行 为研究 [J]. 公路交通科技, 2010, 27(6): 107-111. [Luan Kun, Juan Zhicai, Zong Fang. Research on commuter's choice behavior between travel mode and trip chain. Journal of Highway and Transportation Research and Development, 2010, 27(6): 107-111. ]

[19] 杨励雅, 王振波. 居住区建成环境对居民出行行为影响 的分层线性模型 [J]. 经济地理, 2019, 39(4): 101-108. [Yang Liya, Wang Zhenbo. Impact of residential built environment on travel behavior based on hierarchical linear model. Economic Geography, 2019, 39(4): 101-108. ]

[20] Shiftan Y, Ben-Akiva M, Proussaloglou K, et al. Activity-based modeling as a tool for better understanding travel behaviour [R]. Paper Presented at the 10th Conference of the International Association of Travel Behavior Research. Lucerne, Switzerland, 2003.

[21] Ma J, Mitchell G, Heppenstall A. Daily travel behavior in Beijing, China: An analysis of workers' trip chains, and the role of socio-demographics and urban form [J]. Habitat International, 2014, 43: 263-273.

[22] Wallace B, Barnes J, Rutherford G S. Evaluating the effects of traveler and trip characteristics on trip chaining, with implications for transportation demand management strategies [J]. Transportation Research Record, 2000, 1718: 97-106.

[23] Noland R B, Thomas J V. Multivariate analysis of tripchaining behavior $[\mathrm{J}]$. Environment \& Planning B: Planning and Design, 2007, 34(6): 953-970.

[24] Chowdhury T, Scott D M. Role of the built environment on trip- chaining behavior: An investigation of workers and non-workers in Halifax, Nova Scotia [J]. Transportation, 2020, 47(2): 737-761.

[25] Cheng L, Chen X W, Yang S. An exploration of the relationships between socioeconomics, land use and daily trip chain pattern among low-income residents $[\mathrm{J}]$. Transportation Planning and Technology, 2016, 39(4): 358369.

[26] Ye X, Pendyala R M, Gottardi G. An exploration of the relationship between mode choice and complexity of trip chaining patterns $[\mathrm{J}]$. Transportation Research Part B: Methodological, 2007, 41(1): 96-113.

[27] Nishii K, Kondo K, Kitamura R. Empirical analysis of trip chaining behavior $[\mathrm{J}]$. Transportation Research Record: Journal of the Transportation Research Board, 1988, 1203: 48-59.

[28] Soltani A, Pojani D, Askari S, et al. Socio-demographic and built environment determinants of car use among older adults in Iran [J]. Journal of Transport Geography, 2018, 68: 109-117. 
[29] 鲜于建川, 隽志才, 朱泰英. 通勤出行时间与方式选择 [J]. 上海交通大学学报, 2013, 47(10): 1601- 1605. [Xianyu Jianchuan, Juan Zhicai, Zhu Taiying. Selection of commute trip timing and mode. Journal of Shanghai Jiaotong University, 2013, 47(10): 1601-1605. ]

[30] 耿纪超, 龙如银, 陈红. 居民出行方式选择影响因素的 研究述评 [J]. 北京理工大学学报(社会科学版), 2016, 18(5): 1-9. [Geng Jichao, Long Ruyin, Chen Hong. A review of the influencing factors of residents' travel mode choice. Journal of Beijing Institute of Technology (Social Sciences Edition), 2016, 18(5): 1-9. ]

[31] Rajamani J, Bhat C R, Handy S, et al. Assessing impact of urban form measures on nonwork trip mode choice after controlling for demographic and level-of-service effects [J]. Transportation Research Record, 2003, 1831: 158-165.

[32] 詹姆斯 - 杰卡德. Logistic 回归中的交互效应 [M]. 缪佳, 译. 上海: 上海人民出版社, 2014. [James Jaccard. Interaction effects in logistic regression. Translated by Miu Jia. Shanghai, China: Shanghai People's Publishing House, 2014. ]

[33] Zhang M. The role of land use in travel mode choice: Evidence from Boston and Hong Kong [J]. Journal of the American Planning Association, 2004, 70(3): 344-360.
[34] 杨敏, 陈学武, 王炜, 等. 通勤出行简单链和复杂链的选 择行为研究 [J]. 武汉理工大学学报(交通科学与工程 版), 2008, 32(2): 191-194. [Yang Min, Chen Xuewu, Wang Wei, et al. Behavior analysis of simple/complex trip chaining. Journal of Wuhan University of Technology (Transportation Science \& Engineering), 2008, 32(2): 191-194. ]

[35] 张华歆, 苏逸飞, 智路平. 交通信息下基于出行链的通 勤出行方式选择行为 [J]. 上海海事大学学报, 2016, 37 (1): 49-54, 64. [Zhang Huaxin, Su Yifei, Zhi Luping. Travel mode choice behavior of commuters based on trip chain and traffic information. Journal of Shanghai Maritime University, 2016, 37(1): 49-54, 64. ]

[36] 丁川. 考虑空间异质性的城市建成环境对交通出行的 影响研究 [D]. 哈尔滨: 哈尔滨工业大学, 2014. [Ding Chuan. Research on impacts of urban built environment on travel accounting for spatial heterogeneity. Harbin, China: Harbin Institute of Technology, 2014. ]

[37] 姚江春, 柴彦威. 上海市民高档服装购物出行的交通方 式特征 [J]. 地域研究与开发, 2007, 26(3): 45-50. [Yao Jiangchun, Chai Yanwei. The characteristic of transportation mode in resident's travel for buying high- class clothes in Shanghai. Areal Research and Development, 2007, 26(3): 45-50. ]

\title{
Impact of the built environment on residents' car commuting based on trip chain
}

\author{
ZHANG Xue ${ }^{1,2}$, ZHOU Suhong ${ }^{1,2^{*}}$, CHEN Fei ${ }^{3}$ \\ (1. School of Geography and Planning, Sun Yat-sen University, Guangzhou 510275, China; \\ 2. Guangdong Provincial Engineering Research Center for Public Security and Disaster, Guangzhou 510275, China; \\ 3. China Academy of Urban Planning \& Design, Shenzhen 518040, Guangdong, China)
}

\begin{abstract}
The impact of urban built environment on residents' travel behavior, especially car commuting, is a basic topic of traffic demand forecasting, which has received considerable research attention. Existing studies mainly focused on single trips and paid less attention to the collaborative decision making under the influence of trip chains. By using the logistic model and detailed activity diary data collected in Guangzhou City in 2017, this study investigated the relationship between urban built environment and car commuting on weekdays from the trip chain perspective. Empirical results indicate that there is an interactive relationship between non-commuting trip in the trip chain and the built environment of the workplace and residence, which synergistically affects residents' commuting modes. On the one hand, the non-commuting trip time (before or after the commuter's trip) make difference in the relationship between the built environment and commuting mode choice. On the other hand, the mutual influence of several built environment factors-residential area, work location, and noncommuting activity-determines the commuting behavior. Therefore, the extension of the results may provide new thoughts for predicting individual traffic behavior and traffic demand based on individual models.
\end{abstract}

Keywords: built environment; car commuting trip; trip chain; interaction 